\title{
BAND GAP PROPERTIES OF PERIODIC TAPERED BEAM STRUCTURE USING TRAVELING WAVE METHOD
}

\author{
Tuanjie Li \\ School of Electromechanical Engineering, Xidian University, Xi'an, China \\ e-mail: tjli888@126.com \\ XIAOFEI MA \\ Xi'an Institute of Space Radio Technology, Xi'an, China \\ QIAN ZHANG, ZuOWEI WANG \\ School of Electromechanical Engineering, Xidian University, Xi'an, China
}

\begin{abstract}
The wave motion equations of a tapered beam with respect to axial, torsional and flexural deformations are deduced including the transmission and waveguide equations. Combining the force equilibrium and displacement coordination conditions at the junction, we obtain the relation between the wavenumber and frequency, and the band gap properties of periodic tapered beam structures by the Bloch theorem. The modeling accuracy and efficiency of the traveling wave method are verified by the finite element method. The band gap properties of periodic tampered and uniform beam structures are analyzed and compared for the same materials and lengths as well as the same volumes.
\end{abstract}

Keywords: band gap, periodic structure, traveling wave method, Bloch theorem, tapered beam

\section{Introduction}

In the field of space technology, the majority of structures are complex structures, such as truss structures, frame structures and honeycomb sandwich plate structures. The basic elements of these structures are rods, beams, and plates, etc. For the convenience of manufacturing, most structures are usually built by these basic elements into periodic structures. The wave-bearing properties of periodic structures are governed by their geometries. Wave motion in periodic structures exhibits characteristic frequency intervals called pass and stop bands over which wave motion can or can not occur, respectively. There may be an opportunity to tailor materials to achieve desired band gap characteristics, such that wave propagation is prevented in specified frequency ranges. Therefore, the research on the band gap properties of periodic structures is of significance for vibration isolation design and control of the actual structures in practical engineering.

The analysis of band gap properties of periodic structures dates back to the investigations by Brillouin (1953). He deduced the relation between frequency and wavenumber to describe band gap properties of periodic structures: electric filters and crystal lattices. While since 1960s, the periodic structures consisting of beam-type elements have been attracting great attention in the mechanics and engineering technologies. Many different methods were applied to study the elastic wave propagation in beam-type periodic structures, including lump mass method in Wang et al. (2005), transfer matrix method in Li and Wang (2005) and Yu et al. (2012), plane wave expansion method in Wang et al. (2007), finite element method in Denys (2009) and Liu and Gao et al. (2007), boundary element method in Li et al. (2013), spectral element method in Wen et al. (2014) and Wu et al. (2015) and reverberation-ray matrix method in Guo and Fang (2013). 
Based on the Euler-Bernoulli theorem, Wen et al. (2005) calculated the band gap properties of flexural waves of periodic binary straight beam structures by the plane wave expansion method and the vibration attenuation spectra of a finite sample by the finite element method. A novel vibration isolation structure is designed by using band gap properties of flexural waves. Doyle (1989) proposed an analyticsl spectral element method to obtain accurate wave solutions in the frequency domain based on the vibration equations.

The existing researches on band gap properties of beam-type periodic structures mainly have two aspects of shortcomings. One shortcoming is that the methods lack enough accuracy or versatility. The spectral element method does not have exact wave solutions for all complex structures. Therefore, it is generally used to address band gap properties of one-dimensional periodic structures. The finite element method discretizes a continuous structure into a structure with limited degrees of freedom, which results in a discrete error. The displacement of each member is described by the specified interpolation function. This simplification causes the interpolation error. Due to these errors, the finite element method has large errors for in dynamical analysis of the structures. The other shortcoming is that all mentioned references are based on uniform beams, rarely based on tapered beams. A tapered beam has changeable stiffness along the axial direction due to variable sectional area. They can improve strength and reduce weight for space applications. In addition, they have changeable flexural wavenumber varying with the sectional area. This paper combines the exact traveling wave method with the Bloch theorem to investigate the band gap properties of tapered periodic structures.

The layout of the paper is as follows. First, the traveling wave model of a tapered beam is established. Then, based on the model and Bloch theorem, the relation between wavenumber and frequency is deduced. Finally, simulations to verify the traveling wave model and analysis of the band gap properties of periodic tapered and uniform beam structures are presented.

\section{Traveling wave model of the tapered beam}

The vibration of structures can be considered as superposition of different frequencies and modes of elastic waves. The Fourier transform of each mode of elastic waves is called a wave mode. The different wave modes are related to different frequencies and coupled by both the transmission relation of a member and the scattering relation of a junction. The dynamical characteristics of the overall structures can be described by assembling all wave modes of members.

\subsection{Traveling wave model of a member}

We define the coordinate systems for a member as shown in Fig. 1. $x^{\prime} y^{\prime} z^{\prime}$ is the global coordinate system, and $x y z$ is the local coordinate system.

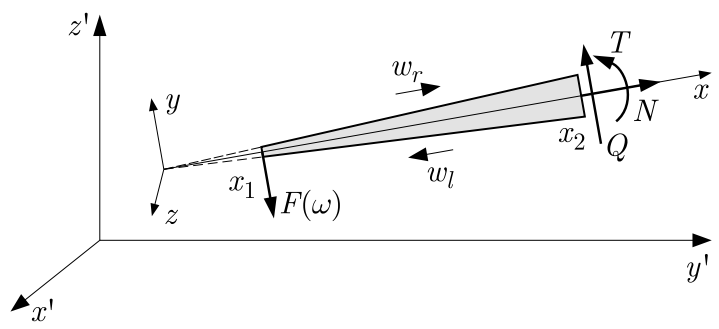

Fig. 1. Coordinate systems of a member

In Fig. $1, w_{l}$ and $w_{r}$ are the left and right traveling wave modes of the member, respectively. In the local coordinate system, the right traveling waves propagate along the $x$-axis in the positive direction. $x_{1}$ and $x_{2}$ are position coordinates of the endpoints of the tapered beam and 
the subscripts 1 and 2 indicate the number of endpoints of the junction. $N$ is the axial force, $T$ is the torsion with respect to $x$-axis, and $Q$ is the transverse force, $F(\omega)$ is the external stimulation.

The displacement and force of the member in the global coordinate system are expressed by

$$
\mathbf{P}=\left\{\begin{array}{l}
\mathbf{U} \\
\mathbf{F}
\end{array}\right\}=\mathbf{M}_{t}\left\{\begin{array}{l}
\mathbf{u} \\
\mathbf{f}
\end{array}\right\}=\mathbf{M}_{t} \mathbf{Y}(x, \omega)\left\{\begin{array}{l}
w_{l} \\
w_{r}
\end{array}\right\}
$$

where $\mathbf{U}$ and $\mathbf{F}$ are the displacement and force vectors in the global coordinate system, and $\mathbf{u}$ and $\mathbf{f}$ are the displacement and force vectors in the local coordinate system, respectively. $\mathbf{M}_{t}$ is the coordinate transformation matrix between the global and local coordinate systems, $\mathbf{Y}(x, \omega)$ is the state transfer matrix and $\omega$ is the frequency of the external stimulation.

The wave modes of the endpoints of the tapered beam are related by a transmission matrix

$$
\left\{\begin{array}{c}
w_{l} \\
w_{r}
\end{array}\right\}_{\mid x_{2}}=\tau\left(x_{2}, x_{1}, \omega\right)\left\{\begin{array}{l}
w_{l} \\
w_{r}
\end{array}\right\}_{\mid x_{1}}
$$

where $\tau\left(x_{2}, x_{1}, \omega\right)$ is the transmission matrix characterizing the variations of the amplitudes and phases of each traveling wave. The waveguide and transmission equations (Eqs. (2.1) and (2.2)) are together called the traveling wave model of the member.

Based on the Euler-Bernoulli beam theory in Riedel and Kang (2006), the specific forms of above equations of the tapered beam are derived in the following.

\subsection{Waveguide equations of the tapered beam}

Generally, the beam structure contains three types of wave modes: axial wave mode, torsional wave mode and flexural wave mode. The thin and straight tapered beam shown in Fig. 1 is assumed to be the ideal elastomer. Then, the length of the beam is $l=x_{2}-x_{1}$, and the sectional area of a tapered beam $A(x)$ is a function of $x$. This function is assumed to be $A(x)=A_{0} x^{2} / a^{2}$, where $a$ is the variation factor of the sectional area, $A_{0}$ is the standard sectional area of the referenced uniform beam in Riedel and Kang (2006).

\subsubsection{Axial waveguide equation}

The force analysis of the tapered beam with respect to stretching and compression deformations is shown in Fig. 2. The force equilibrium equation of an infinitesimal unit is

$$
\rho \frac{1}{2}[A(x+d x)+A(x)] d x \frac{\partial^{2} u}{\partial t^{2}}=\left(N+\frac{\partial N}{\partial x} d x\right)-N=E \frac{\partial A(x) u}{\partial x} d x
$$

where $\rho$ is density, and $u(x, t)$ is the axial displacement.

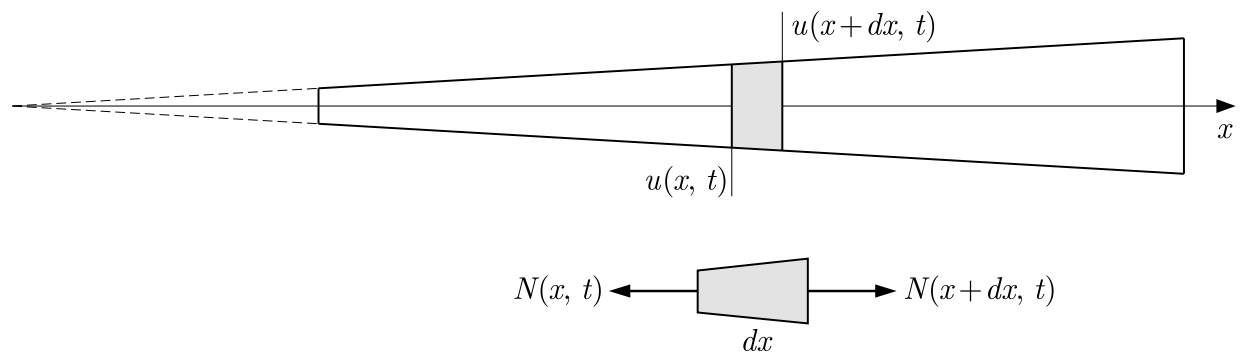

Fig. 2. Axial deformation of the tapered beam

The coefficient of the quadratic differential term of $u$ in Eq. (2.3) must be simplified to obtain analytical wave solutions. We adopt Taylor series expansion of $A(x+d x)$ to obtain the approximation relation 


$$
[A(x+d x)+A(x)] d x \approx 2 A(x) d x+\frac{\partial A(x)}{\partial A(x)} d^{2} x
$$

By omitting the second-order terms of $d x$ in Eq. (2.4), we can further simplify Eq. (2.3) as

$$
\rho A(x) d x \frac{\partial^{2} u(x, t)}{\partial t^{2}}=E A(x) \frac{\partial^{2} u(x, t)}{\partial x^{2}} d x+E \frac{\partial u(x, t)}{\partial x} \frac{d A(x)}{d x} d x
$$

where $E$ is the elasticity modulus. With separation of variables $u(x, t)=\widehat{u}(x, \omega) \mathrm{e}^{\mathrm{j} \omega t}$, the equation of motion of the axial wave is given by

$$
\frac{\partial^{2} \widehat{u}}{\partial x^{2}}+\frac{2}{x} \frac{\partial \widehat{u}}{\partial x}+\frac{\rho \omega^{2}}{E} \widehat{u}=0
$$

The general solution to Eq. (2.6) is

$$
\widehat{u}(x, \omega)=u_{l}+u_{r}=\frac{c_{u l}}{k_{\alpha} x} \exp \left[\mathrm{j}\left(k_{\alpha} x-\frac{\pi}{2}\right)\right]+\frac{c_{u r}}{k_{\alpha} x} \exp \left[-\mathrm{j}\left(k_{\alpha} x-\frac{\pi}{2}\right)\right]
$$

where $u_{l}$ and $u_{r}$ are the undetermined left and right axial traveling wave modes, respectively. The axial wavenumber $k_{\alpha}=\sqrt{\rho \omega^{2} / E}$ depends on the density, elasticity modulus and frequency except the sectional area.

Based on Eq. (2.7), the waveguide equation is expressed by

$$
\left\{\begin{array}{c}
\widehat{u} \\
N
\end{array}\right\}=\left[\begin{array}{cc}
1 & 1 \\
-\frac{E A}{x}+\mathrm{j} k_{\alpha} E A & -\frac{E A}{x}-\mathrm{j} k_{\alpha} E A
\end{array}\right]\left\{\begin{array}{l}
u_{l} \\
u_{r}
\end{array}\right\}
$$

The corresponding transmission equation of wave modes is

$$
\left\{\begin{array}{l}
u_{l} \\
u_{r}
\end{array}\right\}_{\mid x=x_{2}}=\operatorname{diag}\left(\frac{x_{1}}{x_{2}} \mathrm{e}^{\mathrm{j} k_{\alpha} l}, \frac{x_{1}}{x_{2}} \mathrm{e}^{-\mathrm{j} k_{\alpha} l}\right)\left\{\begin{array}{l}
u_{l} \\
u_{r}
\end{array}\right\}_{\mid x=x_{1}}
$$

\subsubsection{Torsional waveguide equation}

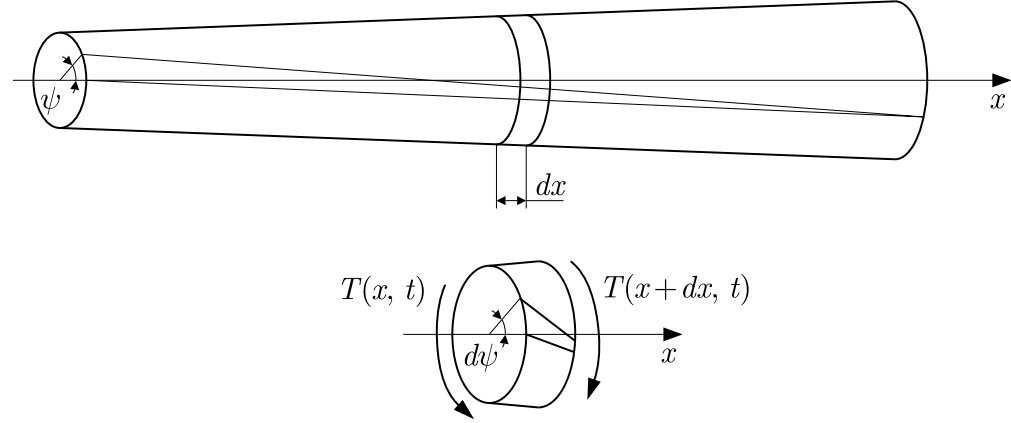

Fig. 3. Torsional deformation of the tapered beam

The torsional motion of the tapered beam is shown in Fig. 3, where $\psi(x, t)$ indicates the angle with respect to the $x$-axis. The moment equilibrium equation of the infinitesimal unit is given as

$$
\begin{aligned}
& \rho I_{p}(x) d x \frac{\partial^{2} \psi(x, t)}{\partial t^{2}}=T(x+d x)-T(x)=\frac{\partial T(x, t)}{\partial x} d x \\
& T=G I_{p} \frac{\partial \psi}{\partial x}
\end{aligned}
$$

where $I_{P}$ is the polar moment of inertia of the section and $G$ is the shear modulus. 
Setting $\psi(x, t)=\phi(x, \omega) \mathrm{e}^{\mathrm{j} \omega t}$, we can obtain the torsional motion equationas follows

$$
\frac{\partial^{2} \phi}{\partial x^{2}}+\frac{4}{x} \frac{\partial \phi}{\partial x}+\frac{\rho \omega^{2}}{G} \phi=0
$$

In a similar way, the waveguide equation of the torsional wave is

$$
\left\{\begin{array}{c}
\phi \\
T
\end{array}\right\}=\left[\begin{array}{cc}
1+\frac{\mathrm{j}}{k_{t} x} & 1-\frac{\mathrm{j}}{k_{t} x} \\
\left(-\frac{3}{x}-\frac{3 \mathrm{j}}{k_{t} x^{2}}+\mathrm{j} k_{t}\right) G I_{P} & \left(-\frac{3}{x}+\frac{3 \mathrm{j}}{k_{t} x^{2}}-\mathrm{j} k_{t}\right) G I_{P}
\end{array}\right]\left\{\begin{array}{l}
\phi_{l} \\
\phi_{r}
\end{array}\right\}
$$

where $\phi_{l}$ and $\phi_{r}$ are the undetermined left and right torsional traveling wave modes, respectively. The torsional wavenumber $k_{t}=\sqrt{\rho \omega^{2} / G}$ is related to the density, shear modulus and frequency, and is independent of the sectional area.

The transmission equation of the torsional wave is described as

$$
\left\{\begin{array}{c}
\phi_{l} \\
\phi_{r}
\end{array}\right\}_{\mid x=x_{2}}=\operatorname{diag}\left(\frac{x_{1}^{2}}{x_{2}^{2}} \mathrm{e}^{\mathrm{j} k_{t} l}, \frac{x_{1}^{2}}{x_{2}^{2}} \mathrm{e}^{-\mathrm{j} k_{t} l}\right)\left\{\begin{array}{c}
\phi_{l} \\
\phi_{r}
\end{array}\right\}_{\mid x=x_{1}}
$$

\subsubsection{Flexural waveguide equation}

The flexural stress analysis of the tapered beam is shown in Fig. 4, where $w_{y}(x, t)$ is the $y$-axis deflection of the beam, $I_{z}=\int_{A} y^{2} d A$ indicates the moment of inertia of the section with respect to the $z$-axis. $M_{z}$ is the bending moment with respect to the $z$-axis and the distribution force is $q=\partial^{2} M_{z} / \partial x^{2}$. The force equilibrium equation of the flexural motion of the infinitesimal element in the plane $x y$ is

$$
\frac{\partial^{2}}{\partial x^{2}}\left(E I_{z} \frac{\partial^{2} w_{y}(x, t)}{\partial x^{2}}\right)=\rho A \frac{\partial^{2} w_{y}(x, t)}{\partial t^{2}}
$$
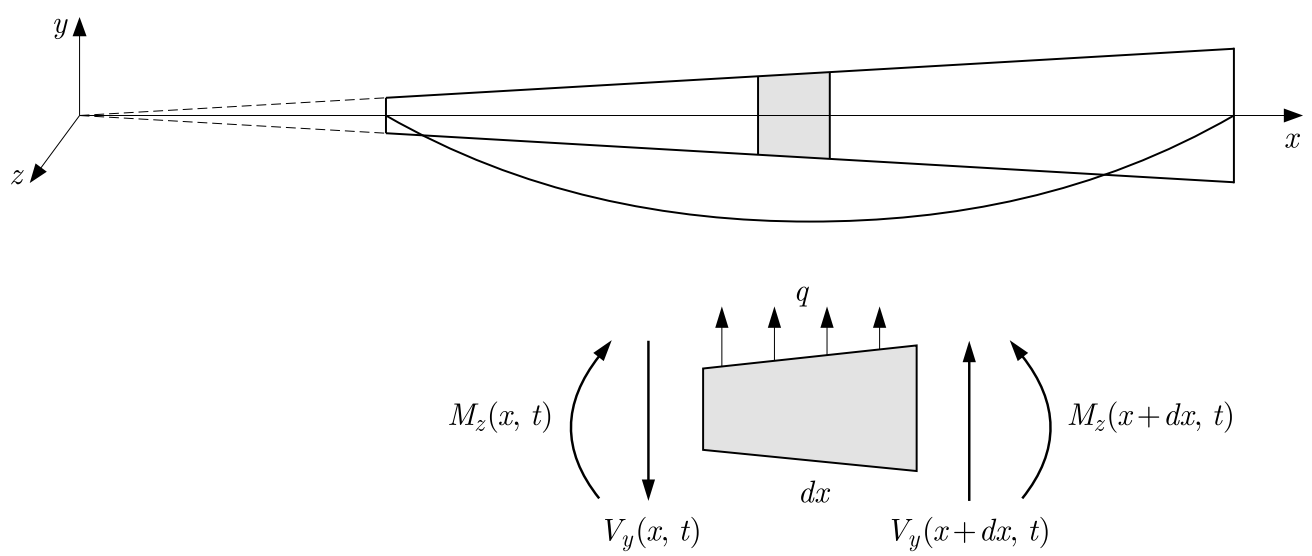

Fig. 4. Flexural deformation of the tapered beam

With the expression of $w_{y}(x, t)=\widehat{w}_{y}(x, \omega) \mathrm{e}^{\mathrm{j} \omega t}$, Eq. (2.14) yields the wave equation of motion

$$
x^{2} \frac{\partial^{4} \widehat{w}_{y}}{\partial x^{4}}+8 x \frac{\partial^{3} \widehat{w}_{y}}{\partial x^{3}}+12 x^{2} \frac{\partial^{2} \widehat{w}_{y}}{\partial x^{2}}-\frac{4 \pi a^{2} \rho \omega^{2}}{E A_{0}} \widehat{w}_{y}=0
$$

The general solution to Eq. (2.15) is

$$
\widehat{w}_{y}=w_{y 1}+w_{y 2}+w_{y 3}+w_{y 4}
$$

where 


$$
\begin{aligned}
w_{y 1} & =\frac{c_{y 1}}{\sqrt[5]{x^{4}}} \exp \left[\mathrm{j}\left(2 k_{z} x-\frac{\pi}{4}\right)\right] & w_{y 2} & =\frac{c_{y 2}}{\sqrt[5]{x^{4}}} \exp \left(2 k_{z} x-\frac{\mathrm{j} \pi}{4}\right) \\
w_{y 3} & =\frac{c_{y 3}}{\sqrt[5]{x^{4}}} \exp \left[-\mathrm{j}\left(2 k_{z} x-\frac{\pi}{4}\right)\right] & w_{y 4} & =\frac{c_{y 4}}{\sqrt[5]{x^{4}}} \exp \left(-2 k_{z} x-\frac{\mathrm{j} \pi}{4}\right)
\end{aligned}
$$

Differently from the wavenumber of axial and torsional wave modes, the wavenumber of the flexural wave mode $k_{z}=\sqrt[4]{\rho A \omega^{2} /\left(E I_{z}\right)}$ not only depends on the density, frequency, elasticity modulus and moment of inertia of area, but also on the sectional area. Therefore, the wavenumber of flexural wave mode of the tapered beam varies with the sectional area. For convenient calculation, the position variable of the section $\mathrm{x}$ is separated from $k_{z}$, i.e. $k_{z}=k_{z}^{\prime} / \sqrt{x}$, where $k_{z}^{\prime}=\sqrt[4]{4 \pi a^{2} \rho \omega^{2} /\left(E A_{0}\right)}$ is a constant.

Then, the waveguide equation of the flexural wave mode is

$$
\left\{\begin{array}{c}
\widehat{w}_{y} \\
\varphi_{z} \\
V_{y} \\
M_{z}
\end{array}\right\}=\left[\begin{array}{cccc}
1 & 1 & 1 & 1 \\
a_{y 1}+\mathrm{j} a_{y 2} & a_{y 1}+a_{y 2} & a_{y 1}-\mathrm{j} a_{y 2} & a_{y 1}-a_{y 2} \\
m_{31} & m_{32} & m_{33} & m_{34} \\
m_{41} & m_{42} & m_{43} & m_{44}
\end{array}\right]\left\{\begin{array}{l}
w_{y 1} \\
w_{y 2} \\
w_{y 3} \\
w_{y 4}
\end{array}\right\}
$$

where $m_{31}=E I_{z}\left(a_{y 3}-\mathrm{j} a_{y 4}-a_{y 5}+\mathrm{j} a_{y 2}^{3}\right), m_{32}=E I_{z}\left(a_{y 3}-a_{y 4}+a_{y 5}-a_{y 2}^{3}\right), m_{33}=E I_{z}\left(a_{y 3}+\right.$ $\left.\mathrm{j} a_{y 4}-a_{y 5}-\mathrm{j} a_{y 2}^{3}\right), m_{34}=E I_{z}\left(a_{y 3}+a_{y 4}+a_{y 5}+a_{y 2}^{3}\right), m_{41}=E I_{z}\left[-\left(9 a_{y 1} /(4 x)\right)-\left(3 \mathrm{j} a_{y 2} / x\right)-a_{y 2}^{2}\right]$, $m_{42}=E I_{z}\left[-\left(9 a_{y 1} /(4 x)\right)-\left(3 a_{y 2} / x\right)+a_{y 2}^{2}\right], m_{43}=E I_{z}\left[-\left(9 a_{y 1} /(4 x)\right)+\left(3 \mathrm{j} a_{y 2} / x\right)-a_{y 2}^{2}\right]$, $m_{44}=E I_{z}\left[-\left(9 a_{y 1} /(4 x)\right)+\left(3 a_{y 2} / x\right)+a_{y 2}^{2}\right]$, and $\varphi_{z}=\partial \widehat{w}_{y} / \partial x$ is the bending angle with respect to the $z$-axis and $V_{y}=E I_{z} \partial^{3} \widehat{w}_{y} / \partial x^{3}$ is the shear force with respect to the $y$-axis. Some coefficients in Eq. (2.17) are $a_{y 1}=-5 /(4 x), a_{y 2}=k_{z}^{\prime} / \sqrt{x}, a_{y 3}=585 /\left(64 x^{3}\right), a_{y 4}=177 k_{z}^{\prime} /\left(16 \sqrt{x^{5}}\right)$, $a_{y 5}=21 k_{z}^{\prime 2} /\left(4 x^{2}\right)$. The transmission equation of the flexural wave modes is

$$
\left\{\begin{array}{c}
w_{l} \\
w_{r}
\end{array}\right\}_{\mid x=x_{2}}=\operatorname{diag}\left(a_{y 6} \mathrm{e}^{2 \mathrm{j} k_{z}^{\prime} a_{y 7}}, a_{y 6} \mathrm{e}^{2 k_{z}^{\prime} a_{y 7}}, a_{y 6} \mathrm{e}^{-2 \mathrm{j} k_{z}^{\prime} a_{y 7}}, a_{y 6} \mathrm{e}^{-2 k_{z}^{\prime} a_{y 7}}\right)\left\{\begin{array}{c}
w_{l} \\
w_{r}
\end{array}\right\}_{\mid x=x_{1}}
$$

where $w_{l}=\left\{w_{y 1}, w_{y 2}\right\}^{\mathrm{T}}, w_{r}=\left\{w_{y 3}, w_{y 4}\right\}^{\mathrm{T}}, a_{y 6}=\sqrt[4]{x_{1}^{5} / x_{2}^{5}}, a_{y 7}=\sqrt{x_{2}}-\sqrt{x_{1}}$.

Due to flexural deformation of the beam with the circular cross-section, in the plane $x y$ and $x z$ there is rotational symmetry with respect to $x$-axis. Similarly, $k_{y}=k_{y}^{\prime} / \sqrt{x}$, where $k_{y}^{\prime}=\sqrt[4]{4 \pi a^{2} \rho \omega^{2} /\left(E A_{0}\right)}$ is a constant. The waveguide equation and the transmission equation of wave modes in the plane $x z$ are obtained just replacing $I_{z}$ and $k_{z}$ by $I_{y}$ and $k_{y}$.

\subsubsection{Spatial beam element}

As the wave motion couples the axial, torsional and flexural wave modes, the corresponding $\mathbf{u}, \mathbf{f}, \mathbf{w}_{l}$ and $\mathbf{w}_{r}$ in Eq. (2.1) can be defined by

$$
\begin{array}{llllll}
\mathbf{u}=\left\{\begin{array}{lllllllll}
\widehat{u} & \widehat{w}_{y} & \widehat{w}_{z} & \phi & \varphi_{y} & \varphi_{z}
\end{array}\right\}^{\mathrm{T}} & \mathbf{f}=\left\{\begin{array}{llllllll}
N & V_{y} & V_{z} & T & M_{y} & M_{z}
\end{array}\right\}^{\mathrm{T}} \\
\mathbf{w}_{l}=\left\{\begin{array}{lllllllll}
u_{l} & w_{y 1} & w_{z 1} & \phi_{l} & w_{y 2} & w_{z 2}
\end{array}\right\}^{\mathrm{T}} & \mathbf{w}_{r}=\left\{\begin{array}{lllllll}
u_{r} & w_{y 3} & w_{z 3} & \phi_{r} & w_{y 4} & w_{z 4}
\end{array}\right\}^{\mathrm{T}}
\end{array}
$$

For the sake of simplicity, the state transfer matrix of tapered beam is described as

$$
Y=\left[\begin{array}{cc}
Y_{u l} & Y_{u r} \\
Y_{f l} & Y_{f r}
\end{array}\right]
$$

where $Y_{u l}, Y_{u r}, Y_{f l}$ and $Y_{f r}$ can be obtained by Eqs. (2.8), (2.12) and (2.17).

The transmission matrix of the spatial tapered beam is obtained as

$$
t\left(x_{2}, x_{1}, \omega\right)=\left[\begin{array}{cc}
t^{\prime}\left(x_{2}-x_{1}\right) & 0 \\
0 & t^{\prime}\left(x_{1}-x_{2}\right)
\end{array}\right]
$$

where $t^{\prime}\left(x_{2}-x_{1}\right)$ and $t^{\prime}\left(x_{1}-x_{2}\right)$ can be founf from Eqs. (2.9), (2.13) and (2.18). 


\section{Band gap properties of periodic tapered beam structure}

The periodic beam structure shown in Fig. 5 is rigidly composed of tapered beam units. Each periodic unit has two tapered beams with different materials rigidly connected together at the junction $\mathbf{H}$. $\mathbf{U}_{n B}^{R}$ and $\mathbf{F}_{n B}^{R}$ indicate the output displacement and force of the $n$-th periodic unit. The superscript $R$ and $L$ denote the right and left endpoints of the tapered beam, respectively. According to the force equilibrium and displacement coordination at the junction, we obtain the following relation of state vectors:

- beam $A$ and beam $B$

$$
\mathbf{F}_{n A}^{R}+\mathbf{F}_{n B}^{L}=\mathbf{0} \quad \mathbf{U}_{n A}^{R}=\mathbf{U}_{n B}^{L}
$$

- periodic units $n$ and $n-1$

$$
\mathbf{F}_{(n-1) B}^{R}+\mathbf{F}_{n A}^{L}=\mathbf{0} \quad \mathbf{U}_{(n-1) B}^{R}=\mathbf{U}_{n A}^{L}
$$

(a)

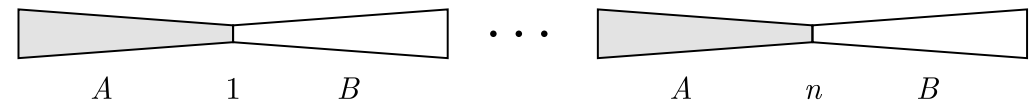

(b)

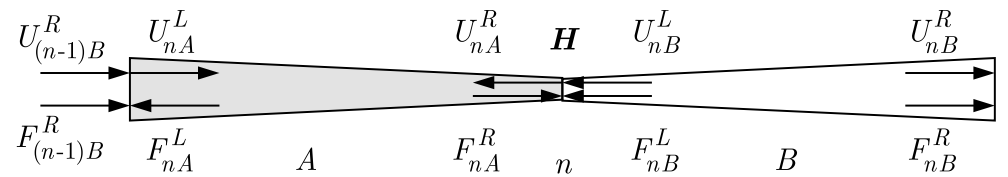

Fig. 5. Periodic tapered beam structure; (a) periodic tapered beam structure, (b) periodic unit

According to the connection relationship of periodic units in Fig. $5, \quad \mathbf{P}_{(n-1) B}^{R}=$ $\left\{\mathbf{U}_{(n-1) B}^{R}, \mathbf{F}_{(n-1) B}^{R}\right\}^{\mathrm{T}}$ is the input state vector of the $n$-th unit, $\mathbf{P}_{n B}^{R}=\left\{\mathbf{U}_{n B}^{R}, \mathbf{F}_{n B}^{R}\right\}^{\mathrm{T}}$ is the output state vector of the $n$-th periodic unit. With Eqs. (3.1) and (3.2), the relationship of state vectors between two tapered beams of the periodic unit and between two periodic units are given by

$$
\mathbf{P}_{n A}^{R}=\operatorname{diag}(\mathbf{I},-\mathbf{I}) \mathbf{P}_{n B}^{L} \quad \mathbf{P}_{(n-1) B}^{R}=\operatorname{diag}(\mathbf{I},-\mathbf{I}) \mathbf{P}_{n A}^{L}
$$

The state vectors $\mathbf{P}_{n i_{2}}^{i_{1}}=\left\{\mathbf{U}_{n i_{2}}^{i_{1}}, \mathbf{F}_{n i_{2}}^{i_{1}}\right\}^{\mathrm{T}}$ and the wave modes vector $\mathbf{W}_{n A}^{i_{1}}=\left\{w_{n l}, w_{n r}\right\}^{\mathrm{T}}$ are defined, where the superscript $i_{1}$ indicates $R$ or $L$ and the subscript $i_{2}$ indicates $A$ or $B$. According to waveguide equation Eq. (2.1) and transmission equation Eq. (2.2), we get

$$
\mathbf{P}_{n i_{2}}^{i_{1}}=\operatorname{diag}(\mathbf{I},-\mathbf{I}) \mathbf{V}_{n i_{2}} \mathbf{Y}_{n i_{2}}^{i_{1}} \mathbf{W}_{n i_{2}}^{i_{1}} \quad \mathbf{W}_{n i_{2}}^{R}=T_{n i_{2}} \mathbf{W}_{n i_{2}}^{L}
$$

where $\mathbf{I}$ is a $3 \times 3$ identity matrix and $\mathbf{V}_{n}$ is the coordinate transformation of the tapered beam $i_{2}$ of the $n$-th periodic unit.

If $m$ is the number of periods, the relation of the input and output state vectors of the $(n+m)$-th periodic unit can be obtained based on Eqs. (3.1)-(3.4)

$$
\begin{aligned}
& \mathbf{P}_{(n+m) B}^{R}=\mathbf{A}_{(n+m-1) B} \mathbf{B}_{(n+m-1) A} \mathbf{P}_{(n+m-1) B}^{R} \\
& \mathbf{A}_{(n+m-1) B}=\mathbf{V}_{(n+m-1) B} \mathbf{Y}_{(n+m-1) B}^{R} \mathbf{T}_{(n+m-1) B}^{L \rightarrow R}\left(\mathbf{Y}_{(n+m-1) B}^{L}\right)^{-1}\left(\mathbf{V}_{(n+m-1) B}\right)^{-1} \\
& \mathbf{B}_{(n+m-1) A}=\mathbf{V}_{(n+m-1) A} \mathbf{Y}_{(n+m-1) A}^{R} \mathbf{T}_{(n+m-1) A}^{R \rightarrow L}\left(\mathbf{Y}_{(n+m-1) A}^{L}\right)^{-1}\left(\mathbf{V}_{(n+m-1) A}\right)^{-1}
\end{aligned}
$$

where the subscript $L \rightarrow R$ indicates the coordinate transformation matrix from the left endpoint to the right endpoint, similarly $R \rightarrow L$. 
The relation between the input state vector of the $n$-th and the output state vector of $(n+m)$-th periodic unit is

$$
\mathbf{P}_{(n+m) B}^{R}=\mathbf{A}_{(n+m-1) B} \mathbf{B}_{(n+m-1) A} \mathbf{P}_{(n+m-1) B}^{R}=\prod_{j=n+m}^{n+1} \mathbf{A}_{(j-1) B} \mathbf{B}_{(j-1) A} \mathbf{P}_{n B}^{R}=\mathbf{C P}_{n B}^{R}
$$

where

$$
\mathbf{C}=\prod_{j=n+m}^{n+1} \mathbf{A}_{(j-1) B} \mathbf{B}_{(j-1) A}
$$

The undetermined state vectors of Eq. (3.6) are much larger than the number of equations. Therefore, Eq. (3.6) is a multiple solutions problem. More equations are needed to obtain the analytical solutions. The state vectors of different periodic units are related by the Bloch theorem. The relation describing the input and output state vectors between the $n$-th and $(n+m)$-th periodic unit by the Bloch theorem is given as

$$
\mathbf{P}_{(n+m) B}^{R}=\operatorname{diag}\left(\mathrm{e}^{\mathrm{j} k m b} \mathbf{I},-\mathrm{e}^{\mathrm{j} k m b} \mathbf{I}\right)=\mathbf{D} \mathbf{P}_{n B}^{R}
$$

where $k$ is the wavenumber of the periodic unit, $b$ is the length of the periodic unit.

Combining Eq. (3.6) with Eq. (3.7), the following expression is obtained

$$
(\mathbf{C}-\mathbf{D}) \mathbf{P}_{n B}^{R}=\mathbf{0}
$$

If the matrix determinant $\operatorname{det}(\mathbf{C}-\mathbf{D})=0$, we can get the relation between the wavenumber and frequency, and the band gap properties.

\section{Numerical simulations}

Two numerical examples are applied to illustrate the proposed method of the tapered beam. The first one is to analyze the vibration response of the tapered cantilever beam with flexural deformations. The veracity and superiority of the proposed method are revealed by comparing with the results of the finite element method. The second one is to compare the differences between the band gap properties of the periodic tapered and uniform beam structure.

\subsection{Dynamic response analysis}

A transverse stimulation applied to the endpoint 1 of the cantilever tapered beam shown in Fig. 1 is $F(\omega)=\mathrm{e}^{\mathrm{j} \omega t}$ and the other endpoint 2 is fixed. The material and geometrical parameters are listed in Table 1.

Table 1. Material and geometrical parameters of the tapered beam

\begin{tabular}{|c|c|c|c|c|c|}
\hline $\begin{array}{c}\text { Elasticity } \\
\text { modulus } E[\mathrm{~Pa}]\end{array}$ & $\begin{array}{c}\text { Density } \\
\rho\left[\mathrm{kg} / \mathrm{m}^{3}\right]\end{array}$ & $\begin{array}{c}\text { Poisson's } \\
\text { ratio } \mu\end{array}$ & $\begin{array}{c}\text { Length } \\
L[\mathrm{~m}]\end{array}$ & $\begin{array}{c}\text { Variation } \\
\text { factor } a\end{array}$ & $\begin{array}{c}\text { Standard } \\
\text { area } A_{0}\left[\mathrm{~m}^{2}\right]\end{array}$ \\
\hline \hline $2.0 \cdot 10^{9}$ & 7800 & 0.3 & 1 & 2 & $5.0 \cdot 10^{-4}$ \\
\hline
\end{tabular}

The frequency response of the transverse displacement of the free end is shown in Fig. 6. The results of the traveling wave model are compared with those of the finite element method. In Fig. 6, the results of the traveling wave method are displayed by the black solid line, and the finite element results obtained by 20 elements and 5 elements per member are shown by the grey dash dot line and black dash line, respectively. 


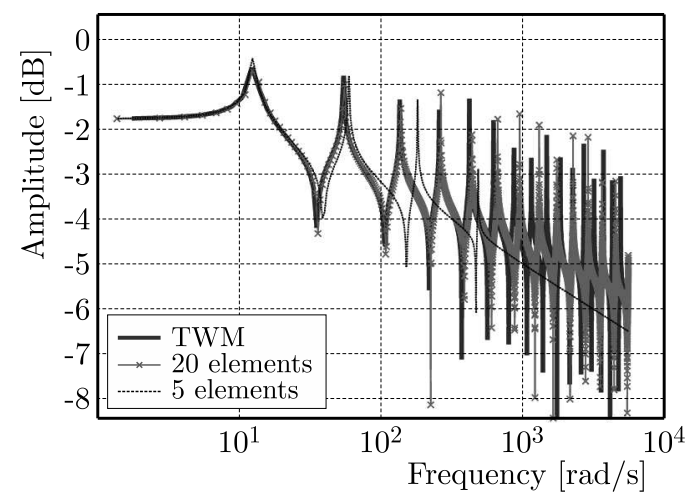

Fig. 6. Frequency response of transverse displacement of the free end

As shown in Fig. 6, the results of the traveling wave model well coincide with the finite element results obtained by 20 elements per member in the low frequency range, which verifies the veracity of the proposed traveling wave method. In the frequency range of $0-1100 \mathrm{rad} / \mathrm{s}$, just 3 resonance peaks are found for finite element results obtained by 5 elements per member. As the number of disperse elements increases, more resonance peaks appear, and the finite element results are converge to the results of the traveling wave method.

\subsection{Band gap analysis}

A periodic unit of the periodic tapered beam structure is shown in Fig. 5. The corresponding material and geometrical parameters are listed in Table 2.

Table 2. Material and geometrical parameters of periodic unit

\begin{tabular}{|l|c|c|c|c|}
\hline \multirow{2}{*}{ Items } & \multicolumn{2}{|c|}{ Tapered beam } & \multicolumn{2}{c|}{ Uniform beam } \\
\cline { 2 - 5 } & $A$ & $B$ & $A$ & $B$ \\
\hline \hline Elastic modulus $E[\mathrm{~Pa}]$ & $8.43 \cdot 10^{9}$ & $1.10 \cdot 10^{11}$ & $8.43 \cdot 10^{9}$ & $1.10 \cdot 10^{11}$ \\
\hline Density $\rho\left[\mathrm{kg} / \mathrm{m}^{3}\right]$ & 1210 & 2730 & 1210 & 2730 \\
\hline Poisson's ratio $\mu[-]$ & 0.3 & 0.3 & 0.3 & 0.3 \\
\hline Length $L[\mathrm{~m}]$ & 0.5 & 0.5 & 0.5 & 0.5 \\
\hline Variation factor $a$ & 2 & 2 & - & - \\
\hline Standard area $A_{0}\left[\mathrm{~m}^{2}\right]$ & $1.59 \cdot 10^{-2}$ & $1.59 \cdot 10^{-2}$ & - & - \\
\hline Sectional area $A\left[\mathrm{~m}^{2}\right]$ & - & - & $6.28 \cdot 10^{-3}$ & $6.28 \cdot 10^{-3}$ \\
\hline
\end{tabular}

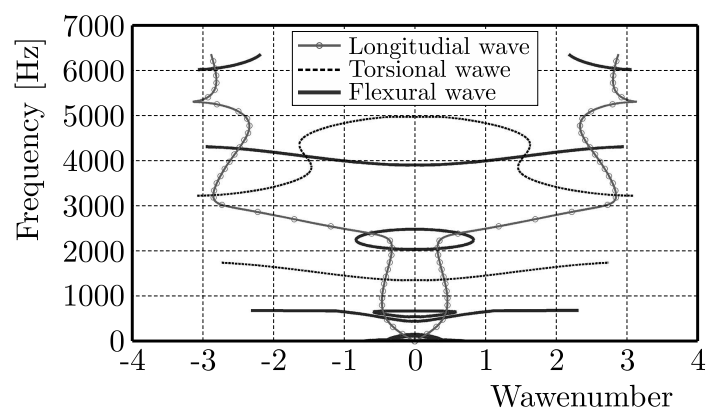

Fig. 7. Band gap properties in three kinds of waves

The band gap properties of the periodic tapered beam structure are shown in Fig. 7. It can be noted that no stop band is found for the axial wave and two stop bands are found for the torsional wave: $0 \mathrm{~Hz}-1353 \mathrm{~Hz}$ and $1758 \mathrm{~Hz}-3229 \mathrm{~Hz}$. The flexural wave has four stop 
bands: $152.9 \mathrm{~Hz}-445.9 \mathrm{~Hz}, 684.7 \mathrm{~Hz}-2038 \mathrm{~Hz}, 2484 \mathrm{~Hz}-3908 \mathrm{~Hz}$ and $4318 \mathrm{~Hz}-6025 \mathrm{~Hz}$. The frequency range $0 \mathrm{~Hz}-152.9 \mathrm{~Hz}$ is the pass band for the axial wave and the flexural wave, but the stop band for the torsional wave, which is called the partial stop band. Other partial stop bands are $152.9 \mathrm{~Hz}-445.9 \mathrm{~Hz}, 684.7 \mathrm{~Hz}-1758 \mathrm{~Hz}, 1758 \mathrm{~Hz}-2038 \mathrm{~Hz}, 2484 \mathrm{~Hz}-3229 \mathrm{~Hz}, 3229 \mathrm{~Hz}-3908 \mathrm{~Hz}$ and $4318 \mathrm{~Hz}-6025 \mathrm{~Hz}$. In the frequency range $152.9 \mathrm{~Hz}-445.9 \mathrm{~Hz}$, only the axial wave contributes to the wavenumber. Therefore, the material or structural parameters of the axial wave can be changed to make it a stop band to prevent wave propagation.

The group velocity of waves is defined as $v=d \omega / d k$. The value of the group velocity represents the speeds of the wave energy transmission. The group velocity curve of the torsional wave is shown in Fig. 8. The group velocity is non-zero in the pass bands $1353 \mathrm{~Hz}-1758 \mathrm{~Hz}$ and $3229 \mathrm{~Hz}-5003 \mathrm{~Hz}$. The group velocity equals to zero in the stop bands $0 \mathrm{~Hz}-1353 \mathrm{~Hz}$ and $1758 \mathrm{~Hz}-$ $3229 \mathrm{~Hz}$, which indicates no energy propagation in the stop bands. It can be observed from Fig. 8 that the group velocity changes with the frequency in the pass band, and the fastest velocity of the energy propagation is at the frequency $3904 \mathrm{~Hz}$. The velocities of the energy transmission in high frequencies are much faster than that in low frequencies.

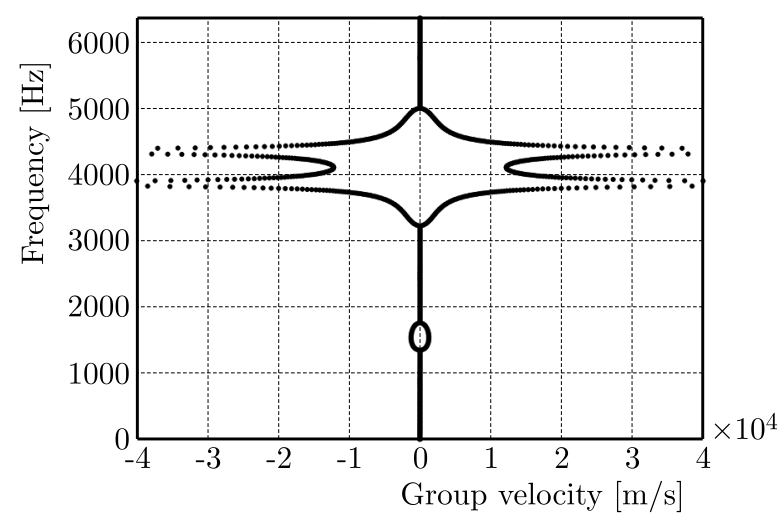

Fig. 8. Group velocity curve of the torsional wave

The material and geometrical parameters of the periodic uniform beam structure are listed in Table 2. The comparisons of band gap properties between the periodic tapered and uniform beam structures with respect to axial, torsional and flexural waves are respectively shown in Fig. 9. The axial wave has three stop bands and the torsional wave has five stop bands. Similarly, the flexural wave has six stop bands. Among these stop bands, no waves can propagate through structure in the frequency ranges $414 \mathrm{~Hz}-675.2 \mathrm{~Hz}, 1143 \mathrm{~Hz}-1341 \mathrm{~Hz}$ and $3322 \mathrm{~Hz}-3723 \mathrm{~Hz}$, which are called the complete stop bands. The other stop bands are the partial stop bands.

As shown in Fig. 9a, the axial wave of the periodic tapered beam structure has no stop bands while that of the periodic uniform beam structure has three stop bands: $1032 \mathrm{~Hz}-2430 \mathrm{~Hz}$, $2975 \mathrm{~Hz}-4634 \mathrm{~Hz}$ and $5357 \mathrm{~Hz}-6010 \mathrm{~Hz}$. It can be inferred that the filter in certain frequency ranges that are complete pass bands for the axial wave and complete stop bands for torsional and flexural waves can be achieved by the periodic tapered beam structure with variational sectional area. Furthermore, the curve slope of the periodic tapered beam structure representing the velocity of energy propagation is bigger than that of the periodic uniform beam structure at the frequency range $0 \mathrm{~Hz}-2000 \mathrm{~Hz}$.

Referring to Fig. 9b, the torsional wave of the periodic tapered beam structure has two stop bands and five stop bands are for the torsional waves of the periodic uniform beam structure: $640.1 \mathrm{~Hz}-1506 \mathrm{~Hz}, 1844 \mathrm{~Hz}-2873 \mathrm{~Hz}, 3322 \mathrm{~Hz}-3726 \mathrm{~Hz}, 4264 \mathrm{~Hz}-4841 \mathrm{~Hz}$ and $5236 \mathrm{~Hz}-6293 \mathrm{~Hz}$. But the stop bands of the periodic tapered beam structure covering a large frequency range are relatively wider than those of the periodic uniform beam structure. It means that the bearing capacity of the torsional force of the periodic tapered beam structure is much better than that 
(a)

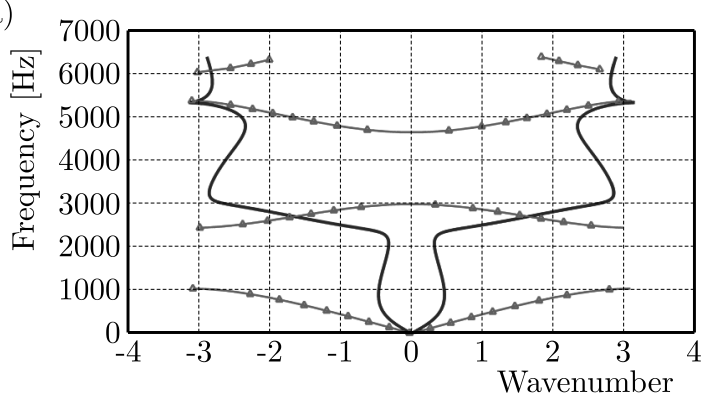

(c)

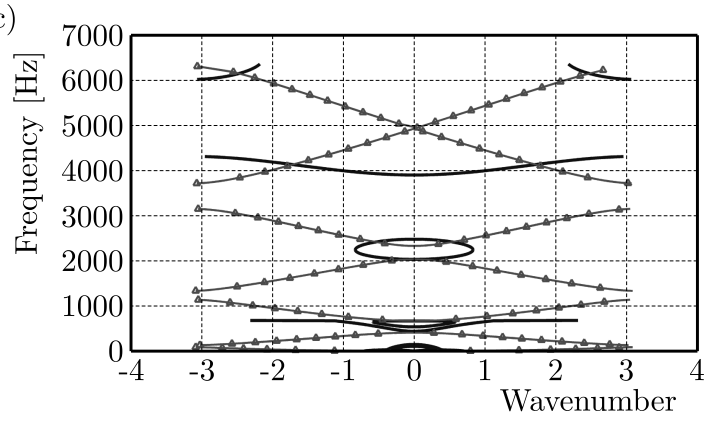

(b)

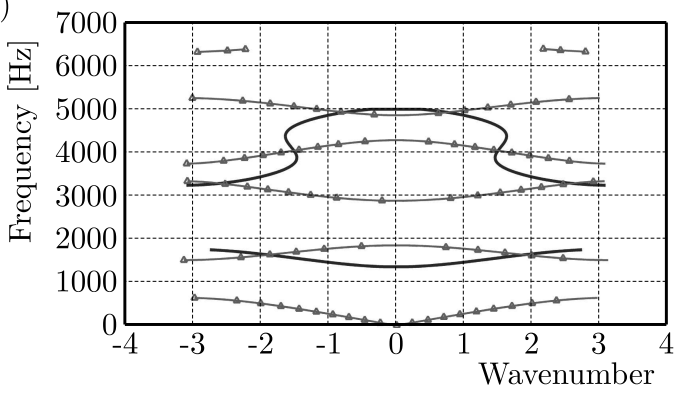

- Tapered beam

$\triangle$ Uniform beam

Fig. 9. Comparison of band gap properties of the: (a) axial wave, (b) torsional wave, (c) flexural wave

of the periodic uniform beam structure. Furthermore, the first stop band of the periodic tapered beam structure covers the whole low frequencies $0 \mathrm{~Hz}-1353 \mathrm{~Hz}$. Therefore, the periodic tapered beam structure is very suitable for vibration isolation of torsional motion.

Referring to Fig. 9c, the frequency range of stop bands of the flexural wave of the periodic uniform beam structure is small. The widest frequency range is just $567 \mathrm{~Hz}$, which is much smaller than that of the periodic tapered beam, $1707 \mathrm{~Hz}$. It means that the bearing capacity of the flexural force of the periodic tapered beam structure is much better than that of the periodic uniform beam structure. The high frequency ranges of stop bands of the periodic tapered beam structure are as approximately the same as those in low frequencies due to the wavenumber changing with the sectional area.

\section{Conclusions}

In this paper, the traveling wave model of the tapered beam is established including the transmission and waveguide equations with respect to the axial, torsional and flexural deformations. Combining these equations with the Bloch theorem, band gap properties of the periodic tapered beam and the periodic uniform beam are analyzed and compared. Some conclusions are summarized as follows.

- Compared with the conventional finite element method, the traveling wave method has higher precision in mid and high frequencies as well as shorter calculation time and less memory occupation. This is because the traveling wave method uses the continuous model to exactly describe the transmission relationship of the member as well as the force equilibrium and displacement coordination conditions of the junction.

- Compared with the uniform beam, the wavenumbers of axial and torsional waves of the tapered beam cannot change with variable sectional area, while the wavenumbers of the flexural wave decrease with an increase in the sectional area.

- The stop bands of the periodic tapered beam structure are wider than those of the periodic uniform beam structure. The periodic tapered beam structure has more advantages over 
the periodic uniform beam structure to achieve vibration isolation and filtering in some frequency ranges.

Acknowledgment

This project is supported by National Natural Science Foundation of China (Grant No. 51375360, U1537213).

\section{References}

1. Brillouin L., 1953, Wave Propagation in Periodic Structures: Electric Filters and Crystal Lattices, McGraw-Hill Book Company Inc., New York

2. DenYs J.M., 2009, The forced vibration of one-dimensional multi-coupled periodic structures: An application to finite element analysis, Journal of Sound and Vibration, 319, 282-304

3. Doyle J.F., 1989, Wave Propagation in Structures: an FFT-Based Spectral Analysis Methodology, Springer-Verlag, New York

4. Guo Y.Q., FAnG D.N., 2013, Analysis and interpretation of longitudinal waves in periodic multiphase rods using the method of reverberation-ray matrix combined with the floquet-bloch theorem, Journal of Vibration and Acoustics, 136, 122-136

5. Li F.L., Wang Y.S., Zhang C.Z., Yu G.L., 2013, Boundary element method for band gap calculations of two-dimensional solid phononic crystals, Engineering Analysis with Boundary Elements, 37, $225-235$

6. Li F.M., Wang Y.S., 2005, Study on wave localization in disordered periodic layered piezoelectric composite structures, International Journal of Solids and Structures, 42, 6457-6474

7. LiU Y., GaO L.T., 2007, Explicit dynamic finite element method for band-structure calculations of 2D phononic crystals, Solid State Communications, 144, 89-93

8. Riedel C.H., Kang B., 2006, Free vibration of elastically coupled dual-span curved beams, Journal of Sound and Vibration, 290, 820-838

9. Wang G., Wen J.H., Wen X.S., 2005, Quasi-one-dimensional phononic crystals studied using the improved lumped-mass method: application to locally resonant beams with flexural wave band gap, Physical Review B, 71, 104302

10. Wang Y.Z., Li F.M., Huang W.H., Wang Y.S., 2007, Effects of inclusion shapes on the band gaps in two-dimensional piezoelectric phononic crystals, Journal of Physics: Condensed Matter, 19, 496204

11. Wen J., Wang G., Yu D.L., Zhao H.G., Liu Y.Z., 2005, Theoretical and experimental investigation of flexural wave propagation in straight beams with periodic structures: Application to a vibration isolation structure, Journal of Applied Physics, 97, 114907-114910

12. Wen S.R., Lu N.L., Wu Z.J., 2014, Dynamic property analysis of the space-frame structure using the spectral element method, Waves Random Complex Medium, 24, 404-420

13. Wu Z.J., Li F.M., ZhANG C., 2015, Vibration band-gap properties of three-dimensional Kagome lattices using the spectral element method, Journal of Sound and Vibration, 341, 162-173

14. Yu D.L., Wen J.H., Shen H.J., XiaO Y., Wen X.S., 2012, Propagation of flexural wave in periodic beam on elastic foundations, Physical Letter A, 376, 626-630 